'Departamento de Ciencias Neurológicas, Facultad de Medicina, Universidad de Chile. Santiago, Chile.

${ }^{2}$ Servicio de Neurología, Neurocirugía y Neurorradiología Intervencional, Clínica Santa María. Santiago, Chile. ${ }^{3}$ Servicio de Neurorradiología, Instituto Neurocirugía Dr. Asenjo. Santiago, Chile.

${ }^{4}$ Corporación de Investigación de Neurología de Santiago. Santiago, Chile.

aPrograma de formación en Neurología, Facultad de Medicina, Universidad de los Andes. Santiago, Chile. ${ }^{\mathrm{b}} \mathrm{PhD}$.

Recibido el 20 de diciembre de 2017, aceptado el 23 de mayo de 2018.

Correspondencia a: Pablo Reyes $\mathrm{S}$. Departamento de Ciencias Neurológicas, Facultad de Medicina, Universidad de Chile. Santiago, Chile. preyes@cinsan.org

\section{Experiencia en el tratamiento endovascular del accidente cerebrovascular isquémico agudo en un centro chileno}

\author{
PABLO REYES S..$^{1,2,4, b}$, LAUTARO BADILLA O. ${ }^{2,3}$, DANIEL ANDREU ${ }^{1}$, \\ VALENTINA BESA ${ }^{\mathrm{a}}$, RODRIGO RIVERA ${ }^{2,3}$, GABRIEL SORDO $^{2,3}$, \\ GONZALO BUSTAMANTE ${ }^{1,2}$, JUAN A. PASTEN ${ }^{2}$, GABRIEL VARGAS ${ }^{2}$, \\ PAULA SILVA ${ }^{2}$, RODRIGO GUERRERO $^{2}$, WALTER FEUERHAKE ${ }^{1,2,4}$
}

\section{Emergency endovascular management of ischemic stroke. Experience in 104 patients}

Background: Recently, five randomized controlled trials confirmed the efficacy and safety of endovascular treatment with or without intravenous thrombolysis in acute ischemic stroke with large-vessel occlusion. Aim: To report patients with ischemic stroke treated with endovascular methods. Material and Methods: Retrospective analysis of 104 patients aged $61 \pm 15$ years (54\% males) with ischemic stroke who received endovascular treatment at a single medical center between 2009 and 2017. Results: Sixty one percent were treated with intravenous thrombolysis plus endovascular procedures and $39 \%$ with endovascular procedures alone. The median door-to needle time was 61 minutes and door-to femoral puncture was 135 minutes. The median National Institute of Health Stroke Scale (NIHSS) scores on admission, 24 hours later and at discharge were 12, 4 and 1 points, respectively. Middle cerebral artery was occluded in $60 \%$ of cases. Other frequent localizations where distal carotid artery in $17 \%$ and vertebro-basilar artery in 14\%. Thrombolysis in cerebral infarction (TICI) scale flow score after the procedure was 3 or $2 b$ in $58 \%$ of cases and significantly correlated with NIHSS scores at 24 hours and discharge. Fifty percent of patients had a mRankin score $<=1$ and ten patients died (9.6\%). Eight percent had a symptomatic intracerebral hemorrhage. Conclusions: The clinical improvement of these patients 24 hours after the procedure and at discharge demonstrate the effectiveness of endovascular treatment in ischemic stroke. The presence neurologists able to interpret multimodal images at the emergency room, the use of local guidelines, the availability of an experienced neuro-interventional team engaged with the workflow and the use of stent retrievers are strongly associated with good outcomes.

(Rev Med Chile 2018; 146: 708-716)

Key words: Reperfusion; Stroke; Thrombolytic Therapy.
$\mathrm{E}$ n Chile el accidente cerebrovascular isquémico (ACVi) es un importante problema de salud pública, constituyendo en el año 2013 la principal causa de muerte y la segunda causa de mortalidad en grupos de entre 30 y 69 años ${ }^{1}$. Desde el año 1995 se utiliza el alteplase endovenoso como tratamiento del ACVi en fase aguda ${ }^{2}$. Nuestro centro ha sido pionero en el tratamiento del accidente cerebrovascular isquémico en Chile. En el año 1999 Feuerhake, Chamorro y Araya publicaron 
la primera serie de pacientes que sufrían un $\mathrm{ACVi}$ agudo tratados mediante trombolisis endovenosa ${ }^{3}$.

En el año 2015 se demostró el beneficio del tratamiento endovascular principalmente con stent retrievers asociado o no a trombolisis endovenosa (mixta) en pacientes con un ACVi de menos de $6 \mathrm{~h}$ de evolución y evidencia de oclusión proximal de arterias de territorio carotídeo ${ }^{4-8}$. En estos estudios, el uso de esta terapia demostró ser superior a la trombolisis endovenosa ${ }^{2,4,7,8,9}$.

El objetivo de este estudio es describir las características clínicas, radiológicas y desenlace de los pacientes con ACVi que recibieron tratamiento endovascular en Clínica Santa María entre los años 2009 y 2017.

\section{Materiales y Métodos}

Serie de casos descriptiva y retrospectiva que incluye a todos los pacientes con diagnóstico de ACVi mayores de 18 años, de territorio carotídeo o vertebrobasilar, que consultaron en el servicio de urgencia (SU) de Clínica Santa María (CSM) y fueron sometidos a tratamiento endovascular, con o sin tratamiento trombolítico previo entre junio de 2009 y junio de 2017. Este estudio fue aprobado por el Comité de Ética local.

Nuestro protocolo de tratamiento endovascular del ACVi agudo incluye pacientes hasta $12 \mathrm{~h}$ desde el inicio de los síntomas y determinación de oclusión arterial intra o extracraneal mediante tomografía axial computada (TC) y Angio TC de cerebro o resonancia magnética de cerebro (RM) en territorio carotídeo o vertebrobasilar (Figura 1). Las imágenes fueron evaluadas por el neurólogo de urgencia en la estación de trabajo. Descartada una hemorragia cerebral y demostrada una oclusión de un vaso proximal (carótida interna distal $(\mathrm{CI})$, arteria cerebral media (ACM) M1 y M2; arteria cerebral posterior (ACP) P1 y P2; arteria basilar o vertebral) se efectuó uno de dos tratamientos según el tiempo de evolución de los síntomas: a) terapia mixta ( $<4,5$ h de evolución): trombolisis endovenosa seguida de tratamiento endovascular (trombectomía mecánica y/o farmacológica) cuando no existía contraindicación para el uso de trombolítico; b) sólo tratamiento

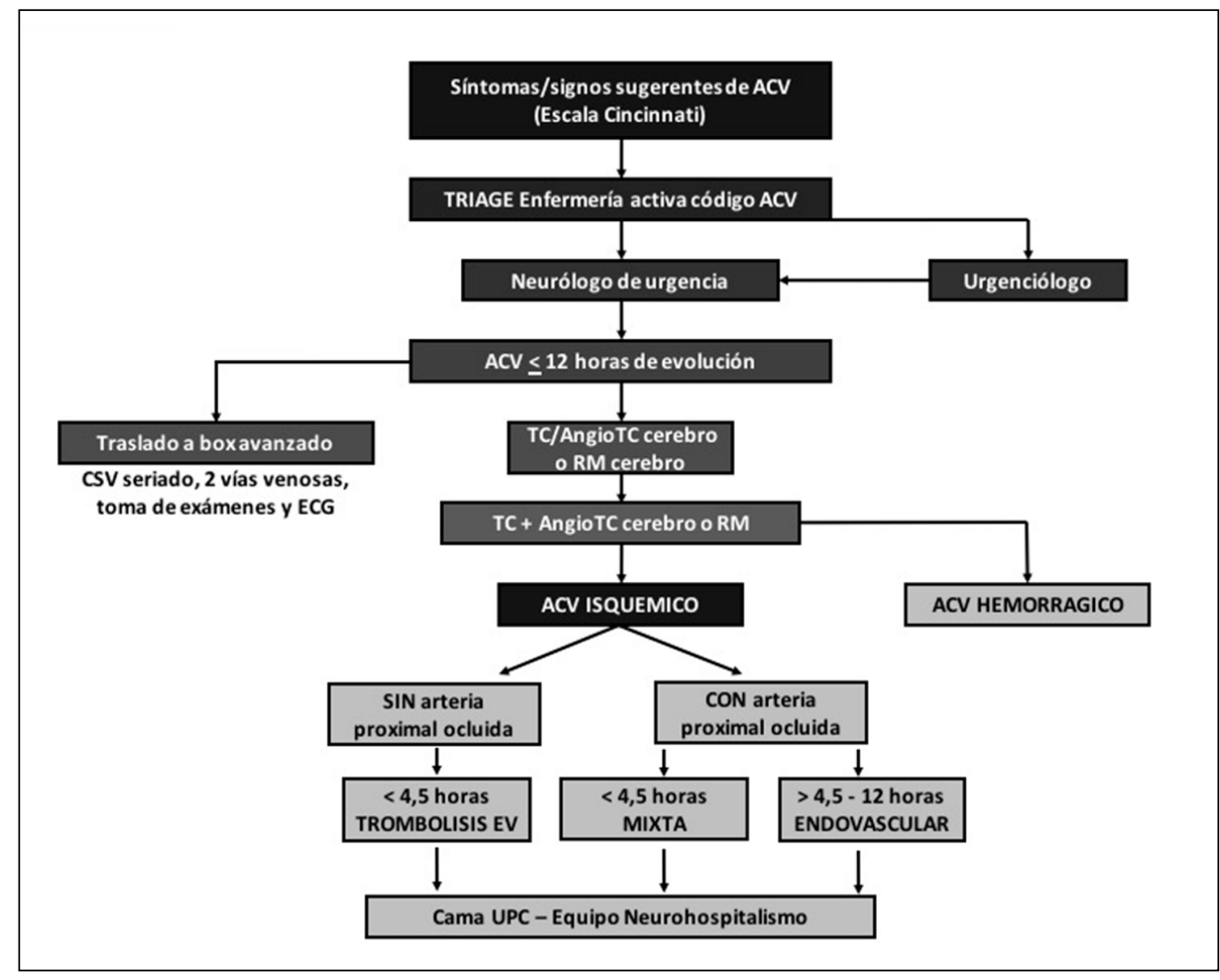

Figura 1. Protocolo de atención de $\mathrm{ACV}$ $<12$ h en CSM. 
endovascular $(>4,5 \mathrm{y}<12 \mathrm{~h}$ de evolución): trombectomía mecánica y/o farmacológica, utilizando para la selección los criterios descritos en la Tabla 1. Para la terapia mixta se administró $0,9 \mathrm{mg} /$ $\mathrm{Kg}$ de r-TPA; $10 \%$ de la dosis durante el primer minuto y el $60 \%$ en $60 \mathrm{~min}$, para administrar el $40 \%$ restante en forma endovascular ${ }^{10}$. A partir del año 2015 administramos $10 \%$ en bolo durante el primer minuto y $90 \%$ de la dosis del r-TPA en 60 $\mathrm{min}^{4}$. Para el tratamiento endovascular farmacológico se utilizó alteplase superselectivo; para la trombectomía mecánica stent retrivers o tromboaspiración. Ocasionalmente se trataron pacientes con lesiones en tándem fuera de los segmentos arteriales descritos.

Para evaluar severidad del ACVi, utilizamos el puntaje en la escala de NIH al ingreso, $24 \mathrm{~h}$ post procedimiento y al alta ${ }^{11}$. Para estimar el grado de discapacidad al alta utilizamos la escala de Rankin modificado (mRS). La etiología fue clasificada según TOAST ${ }^{12}$. Evaluamos retrospectivamente los flujos arteriales post tratamiento endovascular en la angiografía digital de los pacientes entre el año 2012 y 2017 y los clasificamos según TICI ${ }^{13}$.

Tras el procedimiento los pacientes fueron trasladados a una unidad de pacientes críticos y tratados por la unidad de neurología hospitalaria. Para calcular los intervalos de tiempo durante la atención consignamos la hora de inicio de los síntomas reportada por el paciente o sus familiares (inicio), la hora de llegada al SU registrada en la ficha electrónica (puerta), hora de adquisición de la imagen de cerebro (imagen), hora de inicio del tratamiento trombolítico (aguja) y punción en la arteria femoral (punción).

Realizamos un análisis descriptivo expresado como promedio con desviación estándar, mediana con rango intercuartílico y frecuencia (porcen-

\section{Tabla 1. Criterios de inclusión y exclusión para tratamiento mixto y endovascular*}

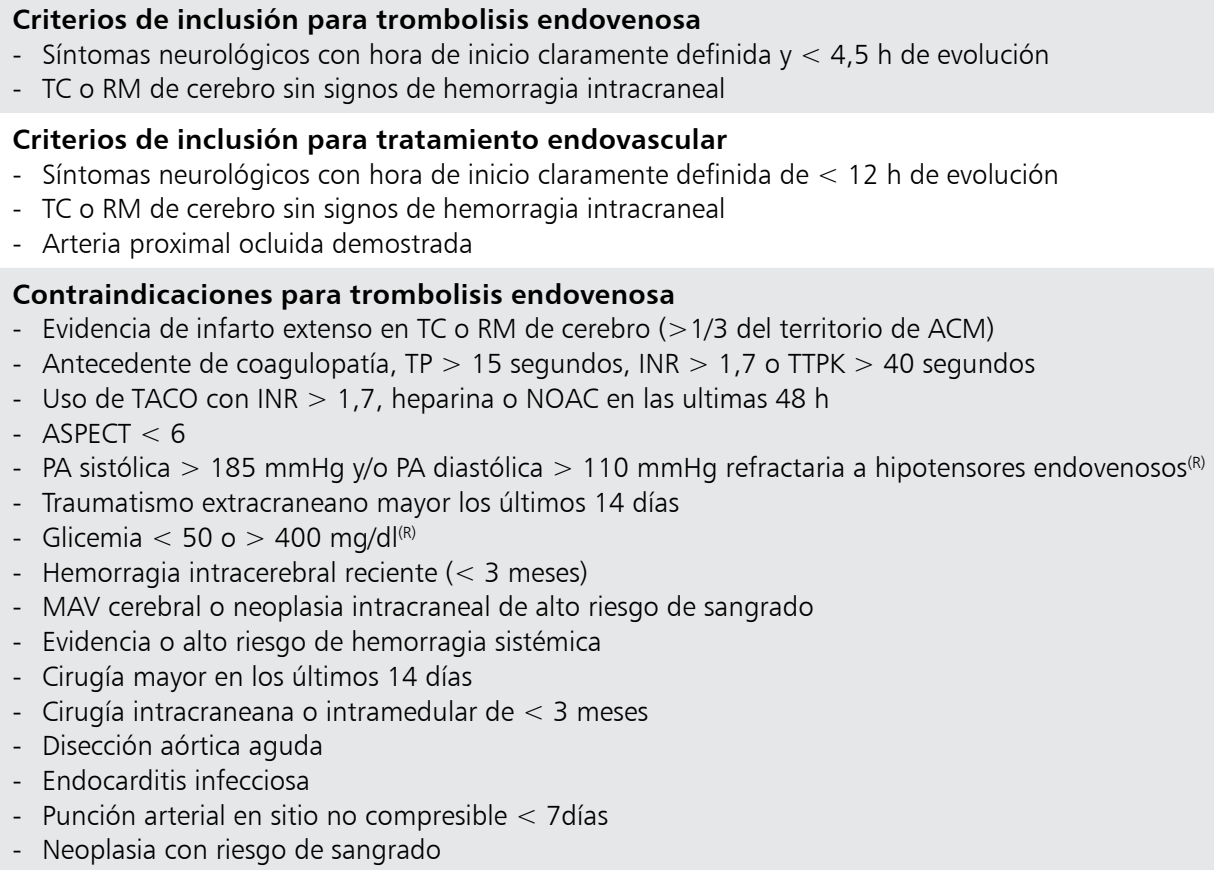

\section{Contraindicaciones para trombolisis endovenosa}

Evidencia de infarto extenso en TC o RM de cerebro ( $>1 / 3$ del territorio de ACM)

Antecedente de coagulopatía, TP $>15$ segundos, INR $>1,7$ o TTPK $>40$ segundos

Uso de TACO con INR $>1,7$, heparina o NOAC en las ultimas $48 \mathrm{~h}$

ASPECT $<6$

PA sistólica $>185 \mathrm{mmHg}$ y/o PA diastólica $>110 \mathrm{mmHg}$ refractaria a hipotensores endovenosos ${ }^{(R)}$

Traumatismo extracraneano mayor los últimos 14 días

Glicemia $<50$ o $>400 \mathrm{mg} / \mathrm{dl}^{(\mathrm{R})}$

Hemorragia intracerebral reciente $(<3$ meses)

MAV cerebral o neoplasia intracraneal de alto riesgo de sangrado

Evidencia o alto riesgo de hemorragia sistémica

Cirugía mayor en los últimos 14 días

Cirugía intracraneana o intramedular de $<3$ meses

Disección aórtica aguda

Endocarditis infecciosa

Punción arterial en sitio no compresible < 7días

Neoplasia con riesgo de sangrado

\section{Contraindicaciones para tratamiento endovascular}

TC con infarto mayor a 1/3 del territorio de ACM

ASPECT $<6$

ACVi $>6$ h y $<12$ h en ausencia de mismatch perfusión-difusión

TACO: tratamiento anticoagulante oral; NOAC: nuevo anticoagulante; ${ }^{(\mathrm{R})}$ Contraindicación relativa. PA: presión arterial. *Estos criterios sufrieron variaciones en el período 2009-2017. 
tajes). Para los análisis de variables paramétricas se utilizó t-test, ANOVA univariado $(\alpha=0,05)$ y corrección de Bonferroni para los análisis post-hoc. En el caso de estadística no paramétrica se utilizó la prueba de Kruskal-Wallis y test de correlación de Spearman.

\section{Resultados}

Entre junio de 2009 y junio de 2017 atendimos 1.875 pacientes con ACVi; 209 de ellos fueron sometidos a tratamiento agudo $(11,1 \%$, rango 7,7-17,3\%), efectuándose 105 trombolisis endovenosas (TEV) $(50,2 \%)$ y 104 tratamientos endovasculares $(49,7 \%)$. Todos los pacientes sometidos a tratamiento endovascular tenían una oclusión de arteria intracraneal. En 64 de ellos $(60,9 \%)$ utilizamos terapia mixta y 40 pacientes $(39,1 \%)$ sólo terapia endovascular.

El 53,8\% de los pacientes fueron hombres $(\mathrm{n}=56)$, con un promedio de edad de 61,1 años (rango 24-90; $\mathrm{DE}=15,8) ; 61,0$ años $(\mathrm{DE}=14,9)$ para los hombres y 71,0 años $(\mathrm{DE}=16,3)$ para las mujeres. El $26,0 \%$ de los pacientes $(\mathrm{n}=27)$ tenía menos de 50 años. El 71,1\% de los pacientes tenían entre 1 y 3 factores de riesgo. En la Tabla 2 se describen los factores de riesgo más prevalentes y la arteria afectada.

La mediana de tiempo desde el inicio de los síntomas a puerta fue de $94 \mathrm{~min}$ (RIC 59-192); mediana puerta-imagen fue de $38 \mathrm{~min}$ (RIC 20-70), puerta-aguja 61 min (RIC 35-107), puerta-punción femoral 135 min (RIC 102-193) (Tabla 2). La mediana de tiempo desde el inicio de los síntomas al comienzo de la trombolisis EV fue de 159 min (RIC 121-230) y a la punción femoral 248 min (RIC 190-332). En el período estudiado hubo una disminución significativa de los tiempos puerta-imagen $\left(F_{(8,88)}=3,70 ; \mathrm{p}=0,001\right)$ y puerta-punción $\left(F_{(8,92)} \stackrel{(8,88)}{=} 4,50 ; \mathrm{p}<0,001\right)$ (Figura 2).

Para decidir el tratamiento se utilizó la combinación de TAC más AngioTAC de cerebro en el $55,8 \%$ de los pacientes $(\mathrm{n}=58)$, en $42,3 \% \mathrm{RNM}$ $(\mathrm{n}=44)$ y en $1,9 \%$ TAC de cerebro $(\mathrm{n}=2)$. Entre el año 2009 y 2012 nuestro protocolo de diagnóstico y selección utilizó prioritariamente RNM y desde el año 2013 a la fecha TAC y AngioTAC de cerebro. La diferencia de los tiempos puerta-imagen entre estos dos períodos fue significativa $(t=4,56$, $\mathrm{df}=95 ; \mathrm{p}<0,001)($ Figura 2).
Tabla 2. Características clínicas, radiológicas y tiempos de atención

\begin{tabular}{|ll|}
\hline Características generales & \\
Edad promedio (años) \pm DE & $61,1(15,8)$ \\
Sexo masculino (\%) & 53,8 \\
mRankin 0-1 antes del ACVi (\%,n) & $95,1(95)$ \\
Antecedentes & \\
Hipertensión arterial (\% pacientes) & 60,6 \\
Dislipidemia (\% pacientes) & 31,7 \\
Tabaquismo (\% pacientes) & 30,8 \\
Fibrilación auricular (\% pacientes) & 24,0 \\
ACV isquémico (\% pacientes) & 22,1 \\
Obesidad (\% pacientes) & 21,2 \\
Diabetes mellitus (\% pacientes) & 14,4 \\
Cardiopatía coronaria (\% pacientes) & 13,5 \\
Puntaje NIH ingreso & \\
General (mediana; RIC) & $12(5-18)$ \\
Tratamiento mixto (mediana; RIC) & $12(6-18)$ \\
Tratamiento endovascular (mediana; RIC) & $12(5-19)$ \\
Arteria ocluida & \\
Cerebral media (\%; n) & $67,3(70)$ \\
Vertebro-basilar (\%; n) & $16,3(17)$ \\
Carótida interna distal (\%; n) & $12,5(13)$ \\
Cerebral posterior (\%; n) & $3,8(4)$ \\
Tipo de tratamiento & \\
Mixto (n; \%) & $64(60,9)$ \\
Endovascular (n; \%) & $40(39,1)$ \\
Tiempos (minutos) & $94(59-192)$ \\
Inicio-puerta (mediana; RIC) & $38(20-70)$ \\
Puerta-imagen (mediana; RIC) & $61(35-107)$ \\
Puerta-aguja (mediana; RIC) & $135(102-193)$ \\
Puerta-punción femoral (mediana; RIC) & $159(121-230)$ \\
Inicio-aguja (mediana; RIC) & $248(190-332)$ \\
Inicio-punción femoral (mediana; RIC) & \\
\hline
\end{tabular}

La mediana de NIH al ingreso fue de 12 puntos (RIC 5-18) (Tabla 2). El 19,2\% de los pacientes $(\mathrm{n}=20)$ puntuaron $\leq 4$ al ingreso a pesar de la oclusión arterial, siendo en 16 de ellos carótida, basilar, M1 o M2. La mediana de NIH a las $24 \mathrm{~h}$ del tratamiento fue de 4 puntos (RIC 1-9) y al egreso fue de 1 (RIC 0-4) (Figura 3). El cambio del puntaje NIH a $24 \mathrm{~h}$ y al egreso resultó significativo $\left(F_{(2,297)}=39,78 ; \mathrm{p}<0,001\right)$. El análisis post-hoc demostró diferencias significativas entre NIH ingreso-24 h; NIH ingreso-egreso y NIH 24 h-egreso (Figura 3). No existe diferencia significativa en la disminución del puntaje $\mathrm{NIH}$ a $24 \mathrm{~h}(\mathrm{t}=-1,68$; $\mathrm{df}=101 ; \mathrm{p}=0,095)$, ni al egreso $(\mathrm{t}=-1,26 ; \mathrm{df}=92$; $\mathrm{p}=0,21)$ entre terapia mixta y sólo endovascular. 

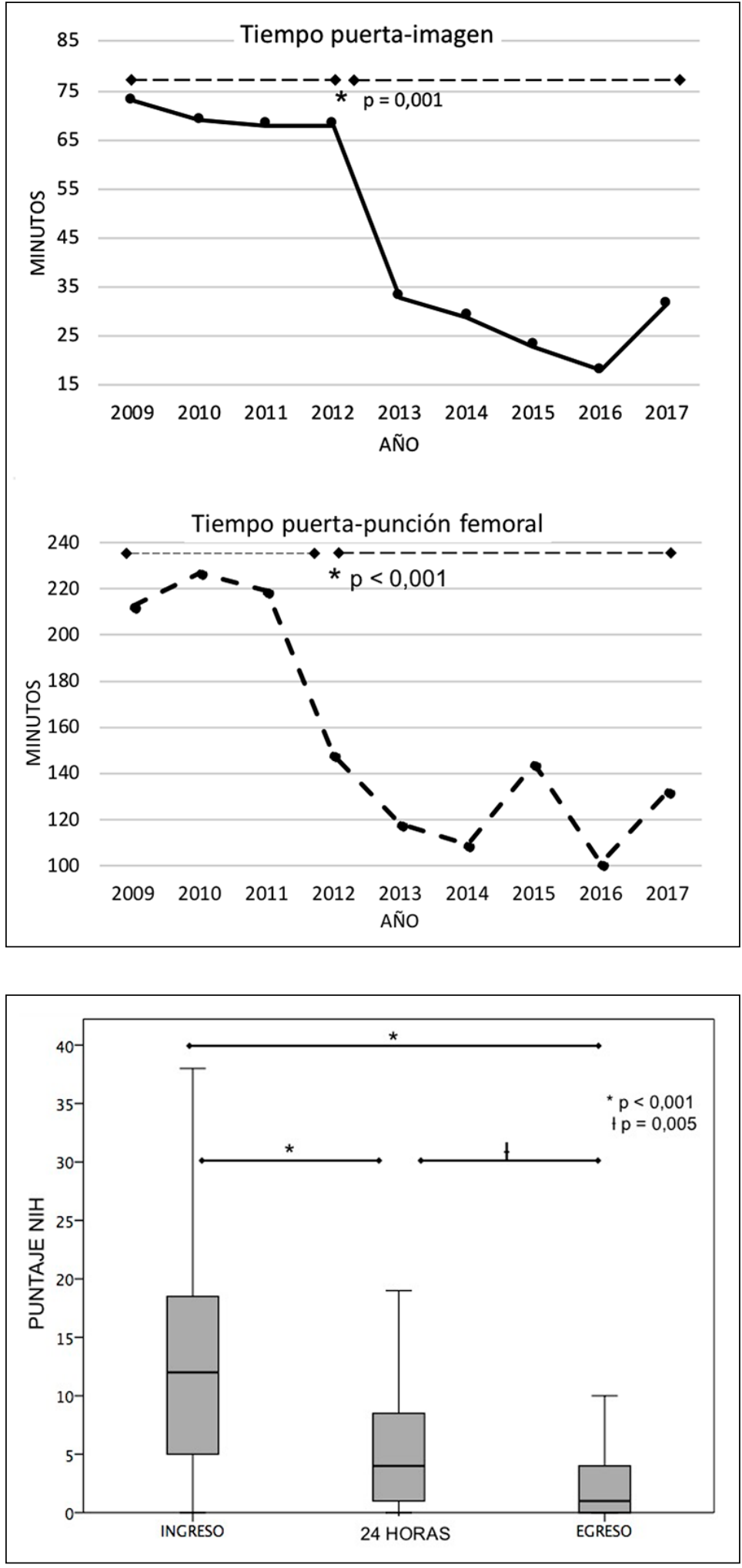

Figura 2. a) Evolución anual del tiempo entre la llegada del paciente y el inicio de la imagen diagnóstica. b) Evolución anual del tiempo entre la llegada del paciente e inicio del tratamiento endovascular. En el período 2009-2017 hubo una disminución significativa de los tiempos de atención en ambos casos.

Figura 3. Box plot del puntaje en la escala $\mathrm{NIH}$ al ingreso, $24 \mathrm{~h}$ de evolución y egreso hospitalario. Al egreso se excluye el $\mathrm{NIH}$ de los pacientes fallecidos, ningún paciente falleció antes de $24 \mathrm{~h}$. 


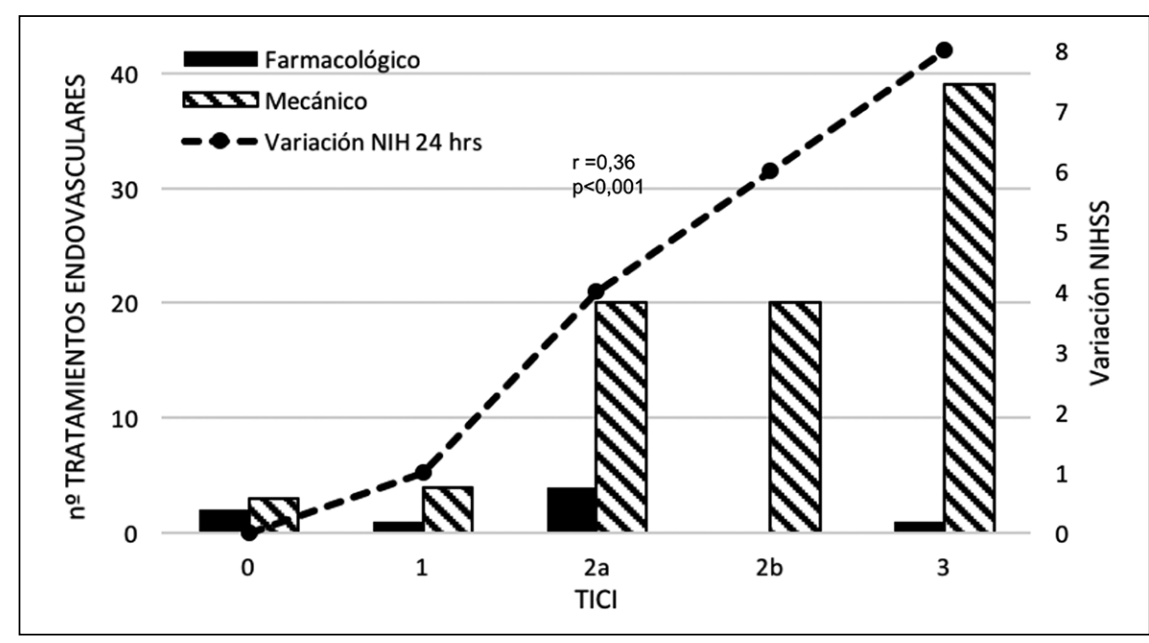

Figura 4. Frecuencias de recanalización postratamiento endovascular según clasificación $\mathrm{TICl}$ y variación del puntaje NIH según grado de reperfusión.
La etiología del ACVi según la clasificación TOAST fue 48,1\% embólicos $(n=50), 20,2 \%$ de causas indeterminadas $(\mathrm{n}=21), 20,2 \%$ otra causa determinada $(\mathrm{n}=21$; fundamentalmente disección arterial $[\mathrm{n}=12])$ y $11,5 \%$ de causa aterotrombótica $(n=12)$. No hubo ACVi lacunares.

En cuanto a la arteria ocluida, fue ACM M1 en $31,7 \%$ de los pacientes $(n=33)$, M2 en $27,9 \%$ $(\mathrm{n}=29)$, M3-M4 en el 2,9\% $(\mathrm{n}=3)$, carótida terminal en $17,3 \%(\mathrm{n}=18)$, vertebrobasilar en $14,4 \%(\mathrm{n}=15)$ y P1-P2 en el 5,7\% $(\mathrm{n}=6)$. En 94 pacientes $(90,3 \%)$ se registró el flujo post recanalización. El 42,5\% $(n=40)$ de estos pacientes fueron clasificados como TICI 3 , el 21,2\% $(\mathrm{n}=20)$ como TICI $2 \mathrm{~b}$ (TICI $2 \mathrm{~b}+3=63,7 \%)$, el 25,5\% $(\mathrm{n}=24)$ como TICI $2 \mathrm{a}$, el 5,3\% $(\mathrm{n}=5)$ como TICI 1 y el $5,3 \%(\mathrm{n}=5)$ como TICI 0 . Se utilizaron stent retrievers en $75 \%(n=78)$ de los procedimientos.

La disminución del puntaje $\mathrm{NIH}$ a las $24 \mathrm{~h}$ según el TICI post tratamiento fue significativa entre pacientes con TICI 3 y TICI $1\left(F_{(4,88)}=3,92\right.$, $\mathrm{p}=0,006 ; \mathrm{rho}=0,36, \mathrm{n}=93, \mathrm{p}<0,001)$ (Figura 4). La disminución del puntaje NIH al egreso según el TICI también fue significativa $\left(F_{(4,79)}=5,81\right.$; $\mathrm{p}<0,0001$; rho $=0,33, \mathrm{n}=83, \mathrm{p}<0,002)$ entre TICI 0 versus el resto; y el TICI 3 versus TICI 0 y 1. No existen diferencias significativas en cuanto al TICI alcanzado según el tipo de tratamiento mixto o sólo endovascular $\left(\chi^{2}=0,009, \mathrm{df}=1\right.$, $\mathrm{p}=0,92)$. Existe una diferencia significativa en el TICI alcanzado según el método endovascular utilizado, siendo más frecuente alcanzar TICI mayores mediante métodos mecánicos $\left(\chi^{2}=8,31\right.$, $\mathrm{df}=1, \mathrm{p}=0,004)$ (Figura 4). Existe una correlación positiva entre el TICI y la variación del puntaje $\mathrm{NIH}$ entre el ingreso y $24 \mathrm{~h}(\mathrm{rho}=0,36 ; \mathrm{p}<0,001)$.

El $7,7 \%$ de los pacientes $(\mathrm{n}=8)$ tuvo una transformación hemorrágica en el TAC o RNM de control a las 24 h y $5,76 \%$ fueron sintomáticas $(\mathrm{n}=6)$. En el transcurso de la hospitalización $9,6 \%$ de los pacientes $(n=10)$ fueron tratados con antibióticos por neumonía y $19,2 \%(\mathrm{n}=20)$ por una infección urinaria baja. Otras complicaciones se describen en la Tabla 3.

Tabla 3. Complicaciones durante la hospitalización

\begin{tabular}{|lrc|}
\hline Complicaciones & \% (n casos) \\
\hline Transformación hemorrágica & 7,69 (8) \\
\hline Transformación hemorrágica sintomática & $5,76 \quad(6)$ \\
\hline Infección tracto urinario bajo & $19,20(20)$ \\
\hline Neumonía & $9,60(10)$ \\
\hline Complicación sitio de punción arterial & $3,80(4)$ \\
\hline Disección carotídea & $0,96(1)$ \\
\hline III par craneal & $0,96(1)$ \\
\hline Otras complicaciones & $11,5 \quad(12)$ \\
- Crisis convulsiva & $1,92(2)$ \\
- Hematuria & $1,92(2)$ \\
- PCR recuperado & $0,96(1)$ \\
- TVP infrapoplítea & $0,96(1)$ \\
\hline
\end{tabular}




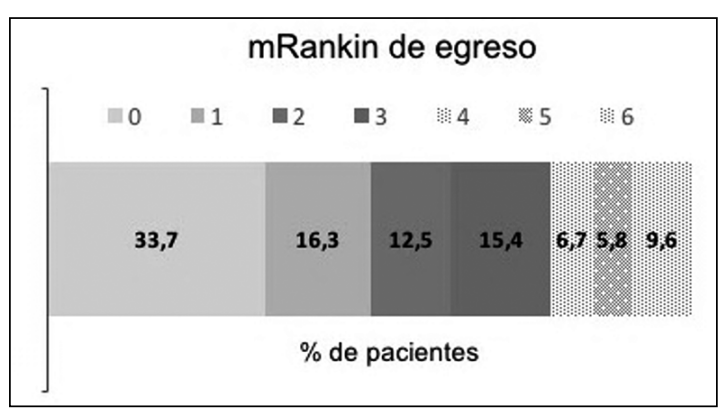

Figura 5. Evaluación de la discapacidad por escala de Rankin modificada (mRankin) al egreso de los 104 pacientes sometidos a tratamiento endovascular.

La mediana de días de hospitalización en nuestro centro fue de 7 días (RIC 5-11). El 73,0\% de los pacientes $(\mathrm{n}=76)$ fue dado de alta desde nuestro centro y el 17,3\% $(\mathrm{n}=18)$ fue trasladado por motivos previsionales. Al alta o traslado el $50,0 \%(n=52)$ se encontraba en mRankin 0 o 1 , el $12,5 \%(n=13)$ en mRankin 2 y $9,6 \%$ de los pacientes falleció (mRankin 6; $\mathrm{n}=10$ ) (Figura 5).

\section{Discusión}

Entre los años 1995 y 2015 la trombolisis endovenosa fue el principal y único tratamiento agudo del ACVi aprobado por la $\mathrm{FDA}^{2}$. En Chile existe experiencia desde el año 1996 con resultados comparables a lo descrito en la literatura ${ }^{3,14-16}$. En el año 2015 una serie de estudios demostraron la eficacia y seguridad del tratamiento endovascular principalmente con stent retrievers precedido o no de terapia endovenosa en territorio carotídeo ${ }^{4-8}$. Seleccionar la mejor terapia para cada paciente es fundamental para lograr una óptima reperfusión del tejido isquémico, en cuanto la probabilidad de recanalización de un vaso de gran calibre, como ACI terminal y segmento M1 de la ACM mediante TEV es baja (4-18\% y $22-33 \%$ respectivamente) ${ }^{17}$, llegando a tasas de recanalización superiores al $70 \%$ con terapias mixtas y sólo endovasculares $^{5,6,18-20}$.

En nuestro centro desde el año 2009 existe residencia neurológica en el servicio de urgencia disponible 24/7. Desde entonces hemos utilizado la visualización de las arterial cerebrales y cervicales como eje del protocolo de atención de pacientes con ACVi agudo. Entre el año 2009 y 2017 hemos actualizado nuestro protocolo de atención de $\mathrm{ACVi}$ en varios aspectos como la selección por imágenes y forma de tratamiento, lo que permitió acortar los tiempos de atención y tratar hasta un $17,3 \%$ de los pacientes con ACVi agudo. Entre los factores de mayor impacto en nuestros resultados está el reemplazo en el año 2013 de la RNM por TAC y AngioTAC por razones estratégicas (Figura 1). Este cambio, sumado a la interpretación de las imágenes por el neurólogo de turno acortó significativamente los tiempos puerta-imagen y puerta-punción femoral (Figura 1). La ausencia de ACVi lacunares confirma la confiabilidad en la interpretación de las imágenes (TAC, AngioTAC y RNM) por neurólogos entrenados en patología cerebrovascular. De los 209 pacientes tratados entre el año 2009 y 2017, el 49,7\% (104 pacientes) tenía una arteria intracraneal proximal obstruida y fueron tratados endovascularmente. De ellos el $60,9 \%$ fue tratado con trombolisis mixta y el $39,1 \%$ sólo con terapia endovascular.

El impacto clínico de estos procedimientos se demuestra en las medianas del puntaje de la escala de NIH pre-tratamiento, 24 h post-tratamiento y al egreso (12, 4 y 1 respectivamente), con una mortalidad de $9,6 \%$ y $50 \%$ de pacientes capaces de realizar vida independiente (mRankin 0-1) al egreso (mediana 7 días). En los estudios MR CLEAN y ESCAPE se reportó una mediana de puntaje NIH a las 24 h de 13 y 6 puntos respectivamente. Estas diferencias se explican por el mayor puntaje NIH de ingreso (17 y 16) en ambos estudios y una mayor frecuencia de obstrucciones en carótida terminal y segmentos M1 o M2 de la $\mathrm{ACM}^{6,18}$. Además, en nuestra serie el $19,2 \%(n=20)$ de los pacientes ingresaron con un puntaje NIH $\leq 4$ a pesar de la obstrucción arterial y el $20,1 \%$ de pacientes sufrió una obstrucción de circulación posterior, territorio que no está fielmente representado en la escala NIH, disminuyendo la mediana de NIH de ingreso y probablemente subestima el beneficio del tratamiento ${ }^{21}$. A pesar de esto, consideramos que la variación en la escala NIH a las $24 \mathrm{~h}$ puede predecir fuertemente la eficacia a largo plazo del tratamiento, sin que requiera esperar el resultado a 90 días medido por escala de mRS, plazo en el que intervienen variables como la rehabilitación, no relacionada con la reperfusión del tejido isquémico, objetivo principal del tratamiento $0^{5,6,22,23}$.

Tras la angiografía logramos flujos post-reca- 
nalización TICI 3 y/o 2 b en el 63,7\% de los casos, lo que se correlacionó positiva y significativamente con la variación del puntaje NIH a 24 h del tratamiento (Figura 3 ) y al egreso. En los estudios Mr. CLEAN y ESCAPE, que incluyeron sólo circulación carotídea, se lograron flujos TICI 3 y/o 2b en el $58,7 \%$ y $72,4 \%$ de los pacientes respectivamente ${ }^{6,18}$. En nuestro centro utilizamos métodos mecánicos desde el año 2009, principalmente stent retrievers $(78 \%$ ) y consideramos que su uso, sumado a una buena selección de los pacientes y experiencia del intervencionista incrementan la probabilidad de un buen resultado. Nuestro protocolo incluye pacientes con oclusiones vertebrobasilares y casos seleccionados de arterias distales. Aunque no existen estudios randomizados de terapia endovascular versus trombolisis endovenosa en estos casos, series de casos han demostrado buenas tasas de recanalización y desenlace funcional ${ }^{24-26}$.

En nuestra serie no encontramos diferencias significativas en la variación del puntaje NIH a las $24 \mathrm{~h}$, ni al egreso según el uso de alteplase endovenoso antes del tratamiento endovascular. A pesar de esto, estimamos beneficioso el uso de terapia mixta por dos razones: 1) la administración de un tratamiento potencialmente favorable en tiempos significativamente menores que el tratamiento endovascular; y 2) estimula la mejora continua de los protocolos para el tratamiento del ACVi en ventana, involucrando activamente al neurólogo en el resto de los cuidados de estos pacientes ${ }^{27}$.

Entre nuestros desafíos más relevantes están el rescate y notificación prehospitalario y la disminución del tiempo imagen-punción femoral. Debemos perfeccionar la selección de pacientes que encontrándose en período de "ventana" no tienen las condiciones para ser sometidos a terapia aguda, así como aquellos que encontrándose fuera de "ventana", cumplen con las condiciones óptimas para ser reperfundidos. El recientemente publicado estudio DAWN demostró beneficios clínicos e imagenológicos de la trombectomía en pacientes seleccionados, con mismatch clínico-radiológico significativo dentro de una "ventana" entre 6 y $24 \mathrm{~h}^{28}$.

Finalmente, hacemos énfasis en que el tratamiento endovascular agudo del ACVi es solo parte de la cadena de atención del ACVi, siendo imprescindibles contar con neurólogos capacitados que garanticen la adecuada evaluación y selección de los pacientes junto al neurorradiólogo intervencional, así como unidades de cuidados neurológicos especializadas y redes de derivación previamente establecidas. Este equipo interdisciplinario entrenado en el cuidado de pacientes con ACV permite mejoras en la calidad de la atención y éxito en el cuidados de éstos y otros pacientes ${ }^{29,30}$.

Agradecimientos: Agradecemos a E.U. Andrea Ampuero C., coordinadora de investigación de la Corporación de Investigación de Santiago (CINSAN), por su invaluable ayuda en la gestión de este proyecto.

\section{Referencias}

1. MINSAL. Ataque Cerebrovascular. 2016. p. http://web. minsal.cl/ataque_cerebral/.

2. The National Institute of Neurological Disorders and Stroke rt-PA Stroke Study Group. Tissue plasminogen activator for acute ischemic stroke. N Engl J Med. 1995; 333 (24): 1581-7.

3. Feuerhake W, Chamorro H, Araya F, W. F, H. C, F. A. Intravenous tissue plasminogen activator in the treatment of acute ischemic stroke: feasibility, safety, and efficiency in the 2 first years of the clinical practice. Act del plasminogeno tisular Intraven en el Trat del infarto Cereb agudo factibilidad, Segur y Efic en los Prim dos anos Pract Clin 1999; 127 (7): 814-9.

4. Berkhemer OA, Fransen PSS, Beumer D, Van Den Berg LA, Lingsma HF, Yoo AJ, et al. A randomized trial of intraarterial treatment for acute ischemic stroke. N Engl J Med 2015; 372 (1).

5. Campbell BCV, Mitchell PJ, Kleinig TJ, Dewey HM, Churilov L, Yassi N, et al. Endovascular Therapy for Ischemic Stroke with Perfusion-Imaging Selection. N Engl J Med 2015; 372 (11): 1009-18.

6. Goyal M, Demchuk AM, Menon BK, Eesa M, Rempel JL, Thornton J, et al. Randomized assessment of rapid endovascular treatment of ischemic stroke. N Engl J Med 2015; 372 (11): 1019-30.

7. Saver JL, Goyal M, Bonafe A, Diener H-C, Levy EI, Pereira VM, et al. Stent-Retriever Thrombectomy after Intravenous t-PA vs. t-PA Alone in Stroke. N Engl J Med 2015; 372 (24): 2285-95.

8. Jovin TG, Chamorro A, Cobo E, de Miquel MA, Molina CA, Rovira A, et al. Thrombectomy within 8 Hours after Symptom Onset in Ischemic Stroke. N Engl J Med 2015; 372 (24): 1-11.

9. Hacke W, Kaste M, Bluhmki E, Brozman M, Dávalos A, Guidetti D, et al. Thrombolysis with Alteplase 3 to 4.5 
Hours after Acute Ischemic Stroke. N Engl J Med 2008; 359 (13): 1317-29.

10. Broderick JP, Palesch YY, Demchuk AM, Yeatts SD, Khatri P, Hill MD, et al. Endovascular Therapy after Intravenous t-PA versus t-PA Alone for Stroke. N Engl J Med 2013; 368 (10): 893-903.

11. Brott T, Adams HP, Olinger CP, Marler JR, Barsan WG, Biller J, et al. Measurements of acute cerebral infarction: a clinical examination scale. Stroke 1989; 20 (7): 864-70.

12. Adams HP, Kappelle LJ, Biller J, Love BB, Gordon DL, Marsh EE. Classification of Subtype of Acute Ischemic Stroke: Definition for Use in a Multicenter Clinical Trial. Stroke 1993; 23 (1): 35-41.

13. Higashida RT, Furlan AJ, Roberts H, Tomsick T, Connors B, Barr J, et al. Trial design and reporting standards for intra-arterial cerebral thrombolysis for acute ischemic stroke. Stroke 2003; 34 (8): e109-37.

14. Figueroa-Reyes T, Sáez MD, Mansilla L E, Sánchez V R, Nogales-Gaete J, Delgado BI. [Thrombolysis for acute ischemic stroke with recombinant tissue plasminogen activator in a Chilean public hospital]. Rev Med Chile 2011; 139 (9): 1118-27.

15. Guevara O C, Bulatova K, Aravena F, Caba S, Monsalve $\mathrm{J}$, Lara $\mathrm{H}$, et al. Trombolisis intravenosa en accidente cerebro vascular isquémico agudo en un hospital público de Chile: Análisis prospectivo de 54 casos. Rev Med Chile 2016; 144 (4): 442-50.

16. Soto VÁ, Morales IG, Grandjean BM, Pollak WD, Del Castillo CC, García FP, et al. [Intravenous thrombolysis for acute ischemic stroke. A four years-experience in a Chilean public hospital]. Rev Med Chile 2017; 145 (4): 468-75.

17. Mortimer AM, Bradley MD, Renowden SA. Endovascular therapy in hyperacute ischaemic stroke: history and current status. Interv Neuroradiol 2013; 19 (4): 506-18.

18. Fransen PSS, Berkhemer OA, Lingsma HF, Beumer D, van den Berg LA, Yoo AJ, et al. Time to Reperfusion and Treatment Effect for Acute Ischemic Stroke: A Randomized Clinical Trial. JAMA Neurol 2015;1-7.

19. Mazighi M, Serfaty JM, Labreuche J, Laissy JP, Meseguer E, Lavallée PC, et al. Comparison of intravenous alteplase with a combined intravenous-endovascular approach in patients with stroke and confirmed arterial occlusion (RECANALISE study): a prospective cohort study. Lancet Neurol 2009; 8 (9): 802-9.

20. del Zoppo GJ, Poeck K, Pessin MS, Wolpert SM, Furlan AJ, Ferbert A, et al. Recombinant tissue plasminogen activator in acute thrombotic and embolic stroke. Ann Neurol 1992; 32 (1): 78-86.

21. Nakiri GS, Castro-Afonso LH, Monsignore LM, Dias FA, Alessio-Alves FF, Fabio SRC, et al. Experience on Mechanical Thrombectomy for Acute Stroke Treatment in a Brazilian University Hospital. J Stroke Cerebrovasc Dis 2017; 26 (3): 532-7.

22. Sajobi TT, Menon BK, Wang M, Lawal O, Shuaib A, Williams D, et al. Early trajectory of stroke severity predicts long-term functional outcomes in ischemic stroke subjects. Stroke 2017; 48 (1): 105-10.

23. Linfante I, Dabus G. Predicting outcomes in the era of endovascular therapy. Stroke 2017; 48: 6-7.

24. Phan K, Phan S, Huo YR, Jia F, Mortimer A. Outcomes of endovascular treatment of basilar artery occlusion in the stent retriever era: a systematic review and meta-analysis. J Neurointerv Surg 2016; 8 (11): 1107-15.

25. Nagel S, Kellert L, Möhlenbruch M, Bösel J, Rohde S, Ringleb P. Improved clinical outcome after acute basilar artery occlusion since the introduction of endovascular thrombectomy devices. Cerebrovasc Dis 2013; 36 (5-6): 394-400.

26. Saber H, Narayanan S, Palla M, Saver JL, Nogueira RG, Yoo AJ, et al. Mechanical thrombectomy for acute ischemic stroke with occlusion of the M2 segment of the middle cerebral artery: a meta-analysis. J Neurointerv Surg 2017 Nov 10.

27. Bracard S, Ducrocq X, Mas JL, Soudant M, Oppenheim C, Moulin T, et al. Mechanical thrombectomy after intravenous alteplase versus alteplase alone after stroke (THRACE): a randomised controlled trial. Lancet Neurol 2016; 15 (11): 1138-47.

28. Nogueira RG, Jadhav AP, Haussen DC, Bonafe A, Budzik RF, Bhuva P, et al. Thrombectomy 6 to 24 Hours after Stroke with a Mismatch between Deficit and Infarct. N Engl J Med 2017; NEJMoa1706442.

29. Snow SJ. Stroke and t-PA-Triggering New Paradigms of Care. N Engl J Med 2016; 374 (9): 809-11.

30. Stroke Unit Trialists' Collaboration. How do stroke units improve patient outcomes. Stroke 1997; 28: 213944. 